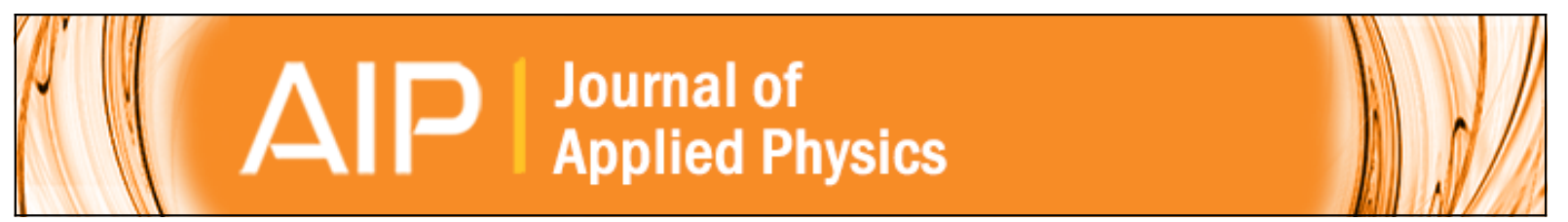

\title{
Quasi-two-dimensional electron gas at the epitaxial alumina/SrTiO3 interface: Control of oxygen vacancies
}

Kristy J. Kormondy, Agham B. Posadas, Thong Q. Ngo, Sirong Lu, Nicholas Goble, Jean Jordan-Sweet, Xuan P. A. Gao, David J. Smith, Martha R. McCartney, John G. Ekerdt, and Alexander A. Demkov

Citation: Journal of Applied Physics 117, 095303 (2015); doi: 10.1063/1.4913860

View online: http://dx.doi.org/10.1063/1.4913860

View Table of Contents: http://scitation.aip.org/content/aip/journal/jap/117/9?ver=pdfcov

Published by the AIP Publishing

\section{Articles you may be interested in}

Growth and transport properties of epitaxial lattice matched half Heusler CoTiSb/InAIAs/InP(001)

heterostructures

Appl. Phys. Lett. 104, 022109 (2014); 10.1063/1.4862191

Oxygen vacancy induced photoluminescence and ferromagnetism in SrTiO3 thin films by molecular beam epitaxy

J. Appl. Phys. 114, 154106 (2013); 10.1063/1.4825257

Optimization of In x Ga 1-x As/In y Al 1-y As high electron mobility transistor structures grown by solid-source molecular beam epitaxy

J. Vac. Sci. Technol. B 19, 490 (2001); 10.1116/1.1359173

Dependence of crystallinity on oxygen pressure and growth mode of La $0.3 \mathrm{Sr} 1.7$ AITaO 6 thin films on different substrates

J. Appl. Phys. 87, 3707 (2000); 10.1063/1.372404

BaTiO3 thin films grown on $\mathrm{SrTiO} 3$ substrates by a molecular-beam-epitaxy method using oxygen radicals J. Appl. Phys. 81, 693 (1997); 10.1063/1.364209

Frustrated by

old technology?

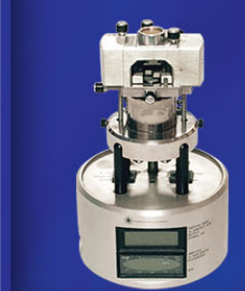

Is your AFM dead

and can't be repaired?

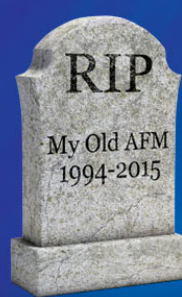

Sick of bad customer support?

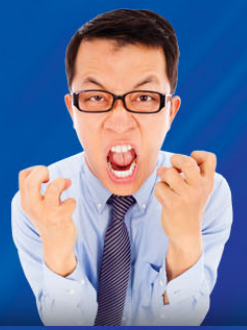

It is time to upgrade your AFM Minimum $\$ 20,000$ trade-in discount for purchases before August 31st

Asylum Research is today's technology leader in AFM 


\title{
Quasi-two-dimensional electron gas at the epitaxial alumina/SrTiO ${ }_{3}$ interface: Control of oxygen vacancies
}

\author{
Kristy J. Kormondy, ${ }^{1}$ Agham B. Posadas, ${ }^{1}$ Thong Q. Ngo, ${ }^{2}$ Sirong Lu, ${ }^{3}$ Nicholas Goble, ${ }^{4}$ \\ Jean Jordan-Sweet, ${ }^{5}$ Xuan P. A. Gao, ${ }^{4}$ David J. Smith, ${ }^{3}$ Martha R. McCartney, ${ }^{3}$ \\ John G. Ekerdt, ${ }^{2}$ and Alexander A. Demkov ${ }^{1, a)}$ \\ ${ }^{1}$ Department of Physics, University of Texas at Austin, Austin, Texas 78712, USA \\ ${ }^{2}$ Department of Chemical Engineering, University of Texas at Austin, Austin, Texas 78712, USA \\ ${ }^{3}$ Department of Physics, Arizona State University, Tempe, Arizona 85287, USA \\ ${ }^{4}$ Department of Physics, Case Western Reserve University, Cleveland, Ohio 44106, USA \\ ${ }^{5}$ IBM T.J. Watson Research Center, Yorktown Heights, New York 10598, USA
}

(Received 9 December 2014; accepted 18 February 2015; published online 5 March 2015)

\begin{abstract}
In this paper, we report on the highly conductive layer formed at the crystalline $\gamma$-alumina/ $\mathrm{SrTiO}_{3}$ interface, which is attributed to oxygen vacancies. We describe the structure of thin $\gamma$-alumina layers deposited by molecular beam epitaxy on $\mathrm{SrTiO}_{3}(001)$ at growth temperatures in the range of $400-800^{\circ} \mathrm{C}$, as determined by reflection-high-energy electron diffraction, $\mathrm{x}$-ray diffraction, and high-resolution electron microscopy. In situ x-ray photoelectron spectroscopy was used to confirm the presence of the oxygen-deficient layer. Electrical characterization indicates sheet carrier densities of $\sim 10^{13} \mathrm{~cm}^{-2}$ at room temperature for the sample deposited at $700^{\circ} \mathrm{C}$, with a maximum electron Hall mobility of $3100 \mathrm{~cm}^{2} \mathrm{~V}^{-1} \mathrm{~s}^{-1}$ at $3.2 \mathrm{~K}$ and room temperature mobility of $22 \mathrm{~cm}^{2} \mathrm{~V}^{-1} \mathrm{~s}^{-1}$. Annealing in oxygen is found to reduce the carrier density and turn a conductive sample into an insulator. (C) 2015 AIP Publishing LLC. [http://dx.doi.org/10.1063/1.4913860]
\end{abstract}

\section{INTRODUCTION}

$\mathrm{SrTiO}_{3}$ (STO) has received much attention because of its large dielectric constant and its role in the integration of other complex oxides on semiconductors, with applications including catalysis, tunable devices, and ferroelectric functionality. ${ }^{1-9}$ A highly interesting application for STO involves the formation of a high mobility two-dimensional electron gas (2DEG) at the oxide/oxide interface. ${ }^{10-30}$ Among the mechanisms for 2DEG formation, one approach involves tailoring an interface between STO and oxides with a large negative enthalpy of formation such as aluminumbased oxides. ${ }^{18-27}$ Under certain conditions, it is energetically favorable for oxygen atoms near the interface to diffuse out of the STO during the initial stages of growth, stabilizing a confined conducting layer. ${ }^{18-27,31,32}$ Since this metallic layer results from the formation of oxygen vacancies near the interface, it characteristically vanishes with oxygen atmospheric annealing. ${ }^{23-27}$

One such heterostructure is alumina/STO. Given the context of archetypical 2DEG $\mathrm{LaAlO}_{3} / \mathrm{STO}$, where competing mechanisms ${ }^{30}$ (including polar catastrophe, ${ }^{10-15}$ cation exchange, ${ }^{16,17}$ and oxygen vacancies ${ }^{18-27}$ ) have been widely investigated and debated, the alumina/STO 2DEG offers an opportunity to isolate the role of oxygen vacancies. Previous studies of crystalline $\gamma-\mathrm{Al}_{2} \mathrm{O}_{3}$ grown by pulsed laser deposition (PLD) have shown that the confinement of the conducting layer depends on growth parameters, particularly substrate temperature. ${ }^{23,24}$ Furthermore, even amorphous oxide heterostructures grown on STO by PLD and atomic layer deposition (ALD) exhibit interfacial conductivity. ${ }^{25-27}$

a)demkov@physics.utexas.edu
Considering that the diffusivity of oxygen in alumina varies widely depending on crystal structure, ${ }^{33,34}$ the properties of the vacancy-controlled STO 2DEG need to be quantified with respect to thin film deposition parameters. The molecular beam epitaxy (MBE) method employed herein facilitates the fabrication of well-ordered oxide heterostructures with precise layer-by-layer atomic control, without complicating factors such as the plume dynamics of PLD ${ }^{23-25}$ or precursor reactivity of ALD. ${ }^{26,27}$ In this paper, we describe the structure of MBE-grown crystalline $\gamma$-alumina on STO (001) as determined by reflection-high-energy electron diffraction (RHEED), x-ray diffraction (XRD), and high-resolution electron microscopy (HREM). The electronic and transport properties of the conducting layer in STO exhibit a strong dependence on the growth temperature, as revealed by x-ray photoelectron spectroscopy (XPS) and electrical measurements.

\section{EXPERIMENTAL DETAILS}

$\mathrm{SrTiO}_{3}(001)$ substrates with dimensions $5 \mathrm{~mm} \times 5 \mathrm{~mm}$ $\times 0.5 \mathrm{~mm}$ (commercially available with $\mathrm{TiO}_{2}$-termination by $\mathrm{HF}$ etching from Crystec) were degreased in acetone, isopropanol, deionized water, and UV ozone. The samples were then introduced into a customized DCA 600 MBE system with a base pressure of $6 \times 10^{-10}$ Torr. More details of the experimental system can be found elsewhere. ${ }^{35,36}$ All substrates were outgassed in the MBE chamber at $700^{\circ}$ for 30 min under ultra-high vacuum (UHV) prior to alumina deposition. The substrate temperature was measured by a thermocouple (calibrated by pyrometer measurement of a silicon substrate) in close proximity to the substrate heater. 
(a)

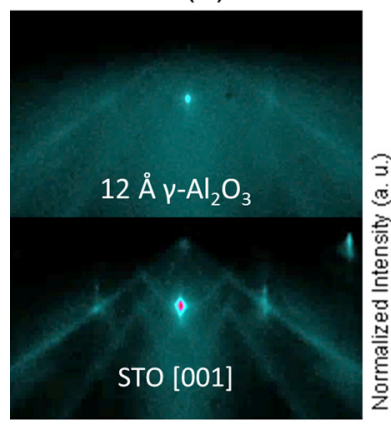

(b)

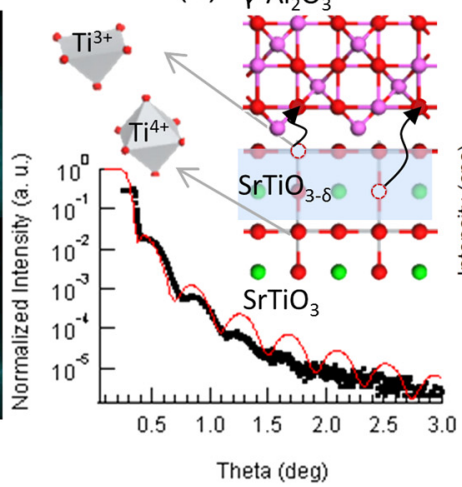

(c)

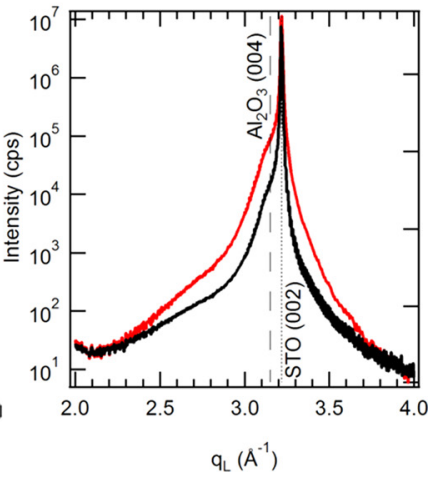

FIG. 1. (a) In situ RHEED of the crystalline STO and alumina surfaces. (b) $\mathrm{X}$-ray reflectivity of structure and interface with inset epitaxial relationship of $\gamma$-alumina on STO (001). (c) Bragg-Brentano scans along the (001) direction at two tilts $\left(\Delta \theta=0.035^{\circ}\right)$.
The substrate temperature during alumina deposition was varied between 400 and $800^{\circ} \mathrm{C}$. Al metal was evaporated from a cold-lip effusion cell with the flux calibrated to have an equivalent $\gamma$-alumina deposition rate of $1.8 \AA$ $/ \mathrm{min}$ as measured by a quartz crystal microbalance. Molecular oxygen was introduced at a background pressure of $1 \times 10^{-6}$ Torr for the growth. The samples were monitored during growth by in situ RHEED. After film deposition, the main shutter was closed, and the samples were cooled down in the presence of oxygen $\left(1 \times 10^{-6}\right.$ Torr $)$.

\section{RESULTS AND DISCUSSION}

STO assumes the cubic $\mathrm{Pm} 3 \mathrm{~m}$ perovskite structure above $110 \mathrm{~K},{ }^{37}$ while the cubic $\gamma$ phase of $\mathrm{Al}_{2} \mathrm{O}_{3}$ is based on the $F d \overline{3} m$ spinel structure with $\mathrm{Al}$ vacancies to satisfy stoichiometry. ${ }^{38,39}$ The measured distances between the diffraction lines in the STO and alumina RHEED patterns (Fig. 1(a)) indicate the lattice relationship represented in Figure 1(b), with an alumina lattice parameter of $7.9 \AA{ }^{38,39}$ The cubic phase of alumina follows the cubic structure of the STO substrate, as the oxygen sublattice of the spinel matches closely that of the perovskite (lattice mismatch $-1 \%$ ). X-ray reflectivity analysis was performed using a Panalytical X'PERT Pro diffractometer (Cu K $\alpha 1$ source, $\lambda=1.5406 \AA$ ) operating at $40 \mathrm{kV}$ and $30 \mathrm{~mA}$. An x-ray reflectivity pattern for a nominally $10-\mathrm{nm}$ alumina/STO sample is also shown in Figure 1(b). Additional $x$-ray diffraction was carried out at the National Synchrotron Light Source beam line X20A (Fig. 1(c)). The low-intensity shoulder to the left of the main STO (002) peak is attributed to alumina. Due to weak scattering from aluminum combined with close proximity to strong STO substrate peaks, strain analysis of the film is not possible; however, in-plane scans (not shown) indicate cubic symmetry with a lattice constant of $7.93 \AA$, consistent with a spinel-based alumina structure. To further characterize the samples, we examined the alumina/STO interface using cross-sectional transmission electron microscopy. Figure 2 shows a representative micrograph recorded with a JEOL JEM-4000EX transmission electron microscope operated at $400 \mathrm{keV}$. This image along the [100] direction clearly reveals an abrupt interface between the highly crystalline epitaxial alumina and the STO substrate. The structural model (inset) shows that the STO substrate is terminated with a $\mathrm{TiO}_{2}$ layer, while the spinel side of the interface commences with aluminum in the 4-fold coordinated tetrahedral site.

XPS measurements were performed in situ using a VG Scienta R3000 analyzer with monochromatic Al K $\alpha$ radiation $(\mathrm{h} \nu=1486.6 \mathrm{eV})$. As illustrated in Figure 3(a), we determine the valence band offset between the STO substrate and a thick (7-nm) alumina film by aligning the spectra such that: (1) the $\mathrm{Sr} 3 d$ core level (CL) in the heterostructure (3$\mathrm{nm}$ alumina/STO) matches that of the pure STO substrate and (2) the $\mathrm{Al} 2 p \mathrm{CL}$ in the thick alumina film matches that of the heterostructure

$$
\begin{aligned}
V B O_{C L}= & \left(E_{S r 3 d}-E_{V B M}\right)_{S T O}-\left(E_{A l 2 p}-E_{V B M}\right)_{A_{2} O_{3}} \\
& -\left(E_{S r 3 d}-E_{A l 2 p}\right)_{A_{2} O_{3} / S T O} .
\end{aligned}
$$

A valence-band offset of $+0.93 \mathrm{eV}$ was calculated and also verified by simulating a thin-film valence band (Fig. 3(b)), as described elsewhere. ${ }^{35}$ As the two methods returned the same value, there is no band bending present. ${ }^{40,41}$ Furthermore, the binding energy of the $\mathrm{Al} 2 p$ core level $(76 \mathrm{eV})$ and the shape of the alumina valence band indicate no sign of any $\mathrm{Al}$ suboxide. ${ }^{40,42}$

During deposition of alumina on STO, two reactions take place: reduction of $\mathrm{STO}^{25,43}$ and oxidation of aluminum metal $^{44,45}$

$$
\mathrm{SrTiO}_{3} \leftrightarrow \mathrm{SrTiO}_{3-x}+\frac{x}{2} \mathrm{O}_{2} \quad\left(\Delta H_{f} \sim x * 250 \mathrm{~kJ} / \mathrm{mol}\right),
$$

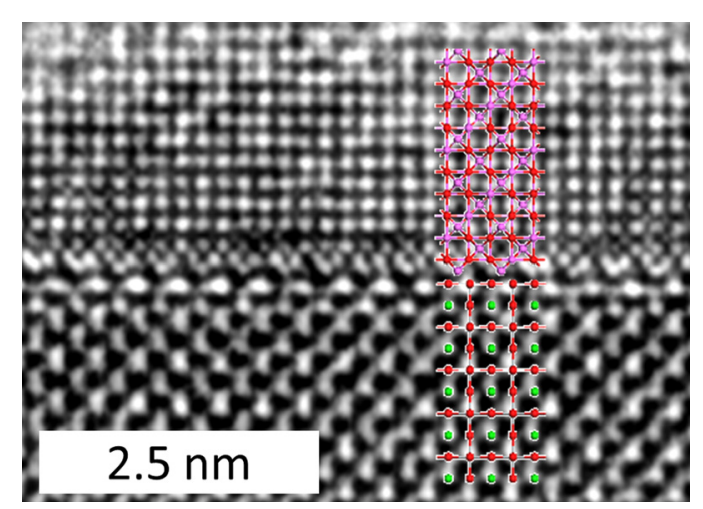

FIG. 2. High-resolution electron micrograph in cross-section geometry confirming abrupt interface between highly crystalline epitaxial alumina on STO substrate. 

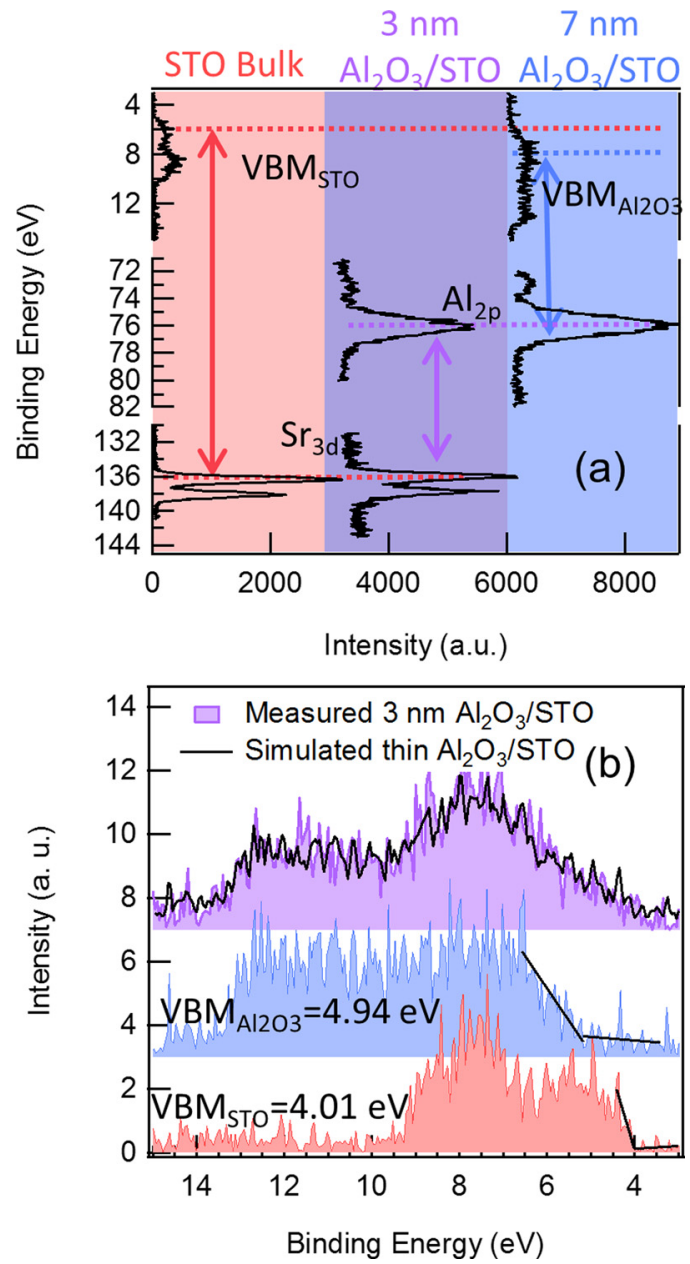

FIG. 3. Core level (a) and valence band (b) spectroscopy for the following: STO substrate (red), $3 \mathrm{~nm}$ alumina/STO (purple), and $7 \mathrm{~nm}$ alumina/STO (blue).

$2 A l+\frac{3-x}{2} O_{2}+\frac{x}{2} O_{2} \leftrightarrow A l_{2} O_{3} \quad\left(\Delta H_{f} \sim-1600 \mathrm{~kJ} / \mathrm{mol}\right)$

As illustrated, reduction of STO provides a fraction of alumina's oxygen, while the remaining fraction originates from oxygen gas (background pressure $10^{-6}$ Torr $\mathrm{O}_{2}$ ). Comparing the enthalpies of formation, it is apparent that even for the extreme case where $100 \%$ of oxygen in alumina comes from STO $(x=3)$, the reaction is thermodynamically favorable. It is also worth noting that the low diffusivity of oxygen in alumina likely suppresses further reduction of STO after completion of the first alumina layer.

The Ti $2 p$ core level provides information on the oxygen vacancy concentration on the STO side of the interface. As illustrated in Fig. 4(a), peak decomposition of the Ti $2 p$ core level in STO allows for a comparison of fully oxidized and reduced Ti. While the bulk STO substrate (purple circles) shows only two spin-split $\mathrm{Ti}^{4+}$ (fully oxidized) peaks, the spectrum after MBE growth of a thin alumina film (raw data: light blue solid line, background: bright blue solid line, fit: black solid line) shows peaks at lower binding energies corresponding to $\mathrm{Ti}$ in a reduced environment $\left(\mathrm{Ti}^{3+}\right.$ : dark blue dashed filled line, $\mathrm{Ti}^{2+}$ : navy dashed filled line) in addition
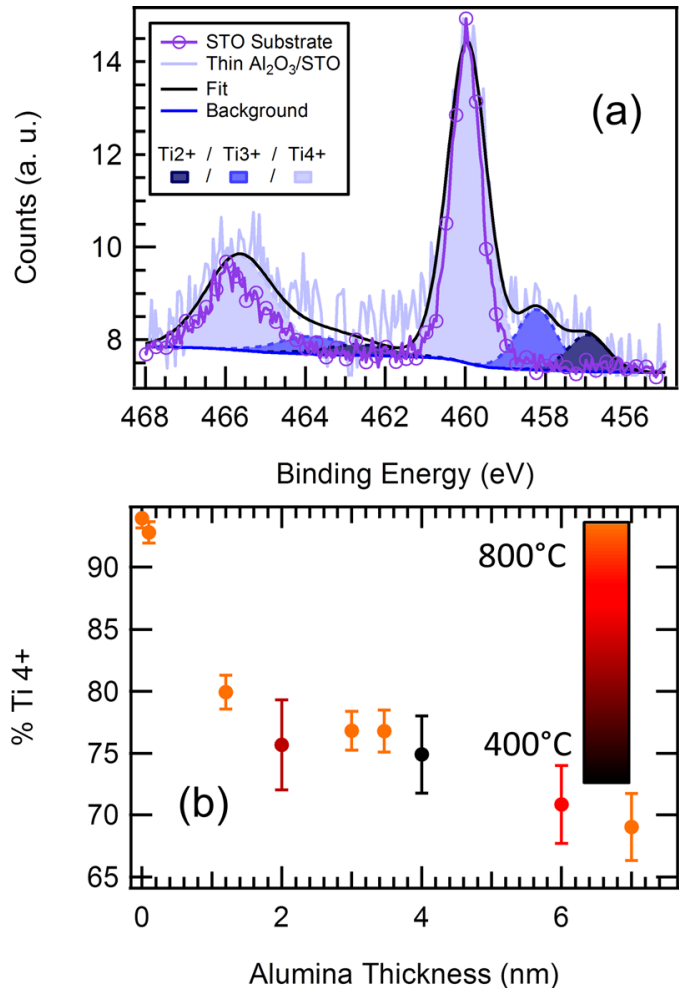

FIG. 4. (a) Peak decomposition of the Ti 2 p core level in STO allows comparison of fully oxidized and reduced Ti. Spectrum for the STO substrate without alumina film is provided for comparison (purple open circles). (b) Analysis of reduced titanium based on XPS Ti 2p spectra for different samples of different thickness and growth temperature.

to the main peak from fully oxidized $\mathrm{Ti}\left(\mathrm{Ti}^{4+}\right.$ : light blue dashed filled line). The percentage of remaining fully oxidized $\mathrm{Ti}^{4+}$ was then calculated by comparing the relative peak areas

$$
C_{4+}=\frac{I\left(T i^{4+}\right)}{I\left(T i^{4+}\right)+I\left(T i^{3+}\right)+I\left(T i^{2+}\right)} .
$$

This analysis of the XPS Ti $2 p$ spectra was repeated for samples of different alumina thicknesses $(0-7 \mathrm{~nm})$ grown at four different substrate temperatures $\left(400-800^{\circ} \mathrm{C}\right.$ in $100{ }^{\circ} \mathrm{C}$ increments), as summarized in Figure 4(b). The resulting depth profile indicates the presence of an interfacial oxygendeficient STO layer.

In order to electrically contact the alumina/STO interface, four indium contacts were placed on scribed corners of each sample in a van der Pauw geometry. Measurements took place in a Quantum Design Physical Property Measurement System (PPMS) capable of applying a $\pm 9 \mathrm{~T}$ magnetic field and 1.9-350 K temperature range. Two Stanford SR830 lockin amplifiers and one SR570 current preamplifier were used to perform a 4-wire electrical transport measurement using less than $1 \mu \mathrm{A}$ current at a frequency of 7 or $13 \mathrm{~Hz}$. Conductivity measurements as a function of temperature using a 4-wire lock-in measurement reveal metallic behavior for the interface between these two insulators (Fig. 5(a)). Comparison between two samples (one deposited at a substrate temperature of $400{ }^{\circ} \mathrm{C}$ and the other at $700^{\circ} \mathrm{C}$ ) indicates a higher sheet resistance for lower growth temperature. 

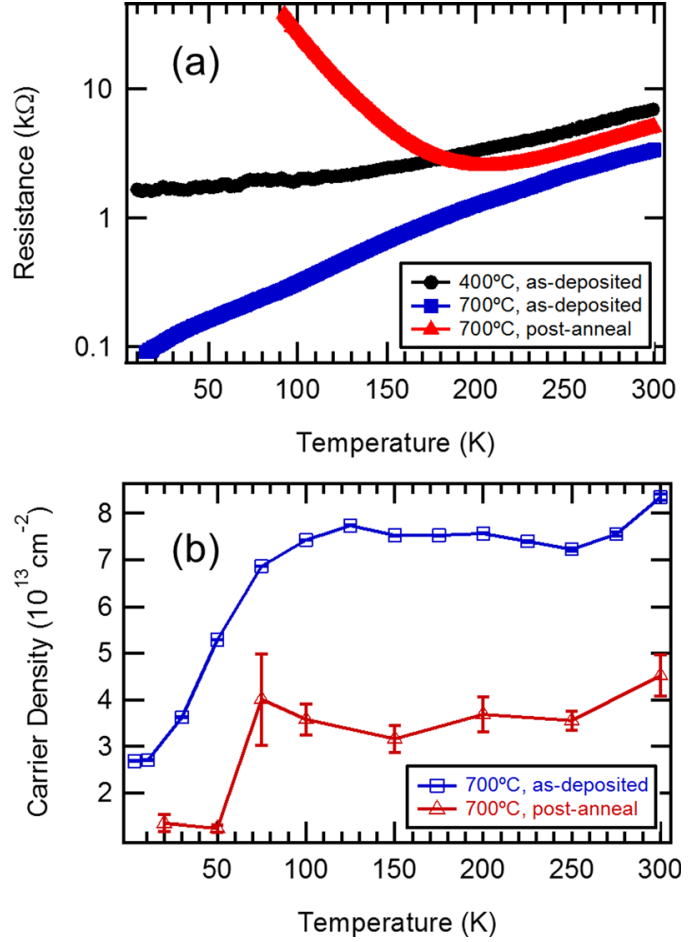

FIG. 5. (a) Sheet resistance of MBE-grown alumina/STO samples grown with deposition temperatures of $700^{\circ} \mathrm{C}\left(6 \mathrm{~nm} \mathrm{Al}_{2} \mathrm{O}_{3}\right)$ and $400{ }^{\circ} \mathrm{C}(4 \mathrm{~nm}$ $\left.\mathrm{Al}_{2} \mathrm{O}_{3}\right)$. Annealing the $700{ }^{\circ} \mathrm{C}\left(6 \mathrm{~nm} \mathrm{Al}_{2} \mathrm{O}_{3}\right)$ sample in 1 Torr of $\mathrm{O}_{2}$ at $400^{\circ} \mathrm{C}$ for 5 min turned the sample's $R(T)$ into an insulating behavior below $200 \mathrm{~K}$. (b). Temperature dependent Hall carrier density of $700{ }^{\circ} \mathrm{C}(6 \mathrm{~nm})$ sample before and after the annealing in $\mathrm{O}_{2}\left(1 \mathrm{Torr}, 400^{\circ} \mathrm{C}\right.$ for $\left.5 \mathrm{~min}\right)$. Hall coefficient was measured using a $\pm 5 \mathrm{~T}$ magnetic field.

Sheet carrier densities on the order of $10^{13}-10^{14} \mathrm{~cm}^{-2}$ are measured in the sample deposited at $700^{\circ} \mathrm{C}$ (Fig. 5(b), blue squares), with a rapid drop in its temperature dependence below $110 \mathrm{~K}$. A maximum electron Hall mobility of $3100 \mathrm{~cm}^{2} \mathrm{~V}^{-1} \mathrm{~s}^{-1}$ at $3.2 \mathrm{~K}$ and room temperature mobility of $22 \mathrm{~cm}^{2} \mathrm{~V}^{-1} \mathrm{~s}^{-1}$ are measured for the heterostructure. For the sample deposited at $400^{\circ} \mathrm{C}$, we were unable to obtain a reliable Hall measurement. However, if one assumes similar carrier density as the sample grown at $700{ }^{\circ} \mathrm{C}$, then the carrier mobility is roughly two times lower at room temperature and ten times lower at $10 \mathrm{~K}$. To elucidate the effect of oxygen vacancy concentration on the carrier density and transport behavior, we annealed the $700^{\circ} \mathrm{C}$ deposited $(6 \mathrm{~nm}$ $\mathrm{Al}_{2} \mathrm{O}_{3}$ ) sample in 1 Torr of $\mathrm{O}_{2}$ at $400^{\circ} \mathrm{C}$ for $5 \mathrm{~min}$. After annealing, the sample showed nearly halved carrier density (Fig. 5(b), red triangles) and insulating behavior at low temperature (Fig. 5(a), red triangles). This attests to the oxygen vacancy origin of carriers at the conductive interface.

\section{CONCLUSION}

In conclusion, we demonstrate the growth of crystalline $\gamma$-alumina on STO using molecular beam epitaxy. X-ray photoelectron spectroscopy of the Ti $2 p$ core level indicates an oxygen-deficient layer at the STO side of the interface. Electrical measurements reveal that the interfacial layer exhibits an increased sheet resistance for decreased growth temperature. Future investigations will study the interface structure in more detail, and its influence on 2DEG mobility and the dimensionality of the conducting layer as determined by Shubnikov-de Hass oscillations. These properties can then be compared with results using other film deposition methods.

\section{ACKNOWLEDGMENTS}

The work at UT and ASU was supported by the Air Force Office of Scientific Research (AFOSR) (FA9550-1210494). The work at CWRU was supported by AFOSR Grant No. FA9550-12-1-0441. Use of the National Synchrotron Light Source, Brookhaven National Laboratory, was supported by the U.S. Department of Energy, Office of Science, Office of Basic Energy Sciences, under Contract No. DE-AC02-98CH10886. Use of facilities in the John M. Cowley Center for High Resolution Electron Microscopy at ASU is also acknowledged.

${ }^{1}$ R. A. McKee, F. J. Walker, and M. F. Chisholm, Phys. Rev. Lett. 81, 3014 (1998).

${ }^{2}$ E. Y. Tsymbal, E. R. A. Dagotto, C.-B. Eom, and R. Ramamoorthy, Multifunctional Oxide Heterostructures (Oxford University Press, 2012).

${ }^{3}$ A. A. Demkov and A. B. Posadas, Integration of Functional Oxides with Semiconductors (Springer, New York, New York, 2014).

${ }^{4}$ S. Hara, M. Yoshimizu, S. Tanigawa, L. Ni, B. Ohtani, and H. Irie, J. Phys. Chem. C 116, 17458 (2012).

${ }^{5}$ C. Hu, K. W. Park, A. Posadas, J. L. Jordan-Sweet, A. A. Demkov, and E. T. Yu, J. Appl. Phys. 114, 183909 (2013).

${ }^{6}$ A. Islam Khan, D. Bhowmik, P. Yu, S. Joo Kim, X. Pan, R. Ramesh, and S. Salahuddin, Appl. Phys. Lett. 99, 113501 (2011).

${ }^{7}$ C. Dubourdieu, J. Bruley, T. M. Arruda, A. Posadas, J. Jordan-Sweet, M. M. Frank, E. Cartier, D. J. Frank, S. V Kalinin, A. A. Demkov, and V. Narayanan, Nat. Nanotechnol. 8, 748 (2013).

${ }^{8}$ C. B. Eom, JOM 49, 47 (1997).

${ }^{9}$ B. T. Liu, K. Maki, Y. So, V. Nagarajan, R. Ramesh, J. Lettieri, J. H. Haeni, D. G. Schlom, W. Tian, X. Q. Pan, F. J. Walker, and R. A. McKee, Appl. Phys. Lett. 80, 4801 (2002).

${ }^{10}$ A. Ohtomo and H. Y. Hwang, Nature 427, 423 (2004).

${ }^{11}$ S. Thiel, G. Hammerl, A. Schmehl, C. W. Schneider, and J. Mannhart, Science 313, 1942 (2006).

${ }^{12}$ N. Nakagawa, H. Y. Hwang, and D. A. Muller, Nat. Mater. 5, 204 (2006).

${ }^{13}$ N. Reyren, S. Thiel, A. D. Caviglia, L. F. Kourkoutis, G. Hammerl, C. Richter, C. W. Schneider, T. Kopp, A.-S. Rüetschi, D. Jaccard, M. Gabay, D. A. Muller, J.-M. Triscone, and J. Mannhart, Science 317, 1196 (2007).

${ }^{14}$ J. Lee and A. A. Demkov, Phys. Rev. B 78, 193104 (2008).

${ }^{15}$ K. Ueno, S. Nakamura, H. Shimotani, A. Ohtomo, N. Kimura, T. Nojima, H. Aoki, Y. Iwasa, and M. Kawasaki, Nat. Mater. 7, 855 (2008).

${ }^{16}$ S. A. Chambers, M. H. Engelhard, V. Shutthanandan, Z. Zhu, T. C. Droubay, L. Qiao, P. V Sushko, T. Feng, H. D. Lee, T. Gustafsson, E. Garfunkel, A. B. Shah, J.-M. Zuo, and Q. M. Ramasse, Surf. Sci. Rep. 65, 317 (2010).

${ }^{17}$ S. A. Chambers, Surf. Sci. 605, 1133 (2011).

${ }^{18}$ A. Kalabukhov, R. Gunnarsson, J. Börjesson, E. Olsson, T. Claeson, and D. Winkler, Phys. Rev. B 75, 121404 (2007).

${ }^{19}$ R. Pentcheva and W. E. Pickett, Phys. Rev. B 74, 035112 (2006).

${ }^{20}$ J. N. Eckstein, Nat. Mater. 6, 473 (2007).

${ }^{21}$ G. Herranz, M. Basletić, M. Bibes, C. Carrétéro, E. Tafra, E. Jacquet, K. Bouzehouane, C. Deranlot, A. Hamzić, J.-M. Broto, A. Barthélémy, and A. Fert, Phys. Rev. Lett. 98, 216803 (2007).

${ }^{22}$ N. Pavlenko, T. Kopp, E. Y. Tsymbal, J. Mannhart, and G. A. Sawatzky, Phys. Rev. B 86, 064431 (2012).

${ }^{23}$ Y. Z. Chen, N. Bovet, F. Trier, D. V. Christensen, F. M. Qu, N. H. Andersen, T. Kasama, W. Zhang, R. Giraud, J. Dufouleur, T. S. Jespersen, J. R. Sun, A. Smith, J. Nygård, L. Lu, B. Büchner, B. G. Shen, S. Linderoth, and N. Pryds, Nat. Commun. 4, 1371 (2013).

${ }^{24}$ Y. Z. Chen, N. Bovet, T. Kasama, W. W. Gao, S. Yazdi, C. Ma, N. Pryds, and S. Linderoth, Adv. Mater. 26, 1462 (2014).

${ }^{25}$ Y. Chen, N. Pryds, J. E. Kleibeuker, G. Koster, J. Sun, E. Stamate, B. Shen, G. Rijnders, and S. Linderoth, Nano Lett. 11, 3774 (2011).

${ }^{26}$ S. W. Lee, Y. Liu, J. Heo, and R. G. Gordon, Nano Lett. 12, 4775 (2012). 
${ }^{27}$ S. W. Lee, J. Heo, and R. G. Gordon, Nanoscale 5, 8940 (2013).

${ }^{28}$ J. Mannhart and D. G. Schlom, Science 327, 1607 (2010).

${ }^{29}$ P. Zubko, S. Gariglio, M. Gabay, P. Ghosez, and J.-M. Triscone, Annu. Rev. Condens. Matter Phys. 2, 141 (2011).

${ }^{30}$ L. Yu and A. Zunger, Nat. Commun. 5, 5118 (2014).

${ }^{31}$ C. Lin and A. A. Demkov, Phys. Rev. Lett. 111, 217601 (2013).

${ }^{32}$ C. Mitra, C. Lin, J. Robertson, and A. A. Demkov, Phys. Rev. B 86, 155105 (2012).

${ }^{33}$ T. Nabatame, T. Yasuda, M. Nishizawa, M. Ikeda, T. Horikawa, and A. Toriumi, Jpn. J. Appl. Phys., Part 1 42, 7205 (2003).

${ }^{34}$ Y. Oishi and W. D. Kingery, J. Chem. Phys. 33, 480 (1960).

${ }^{35}$ K. J. Kormondy, A. B. Posadas, A. Slepko, A. Dhamdhere, D. J. Smith, K. N. Mitchell, T. I. Willett-gies, S. Zollner, L. G. Marshall, J. Zhou, and A. A. Demkov, J. Appl. Phys. 115, 243708 (2014).

${ }^{36}$ M. Choi, A. B. Posadas, C. A. Rodriguez, A. O'Hara, H. Seinige, A. J. Kellock, M. M. Frank, M. Tsoi, S. Zollner, V. Narayanan, and A. A. Demkov, J. Appl. Phys. 116, 043705 (2014).
${ }^{37}$ T. Riste, E. J. Samuelsen, K. Otnes, and J. Feder, Solid State Commun. 9 , 1455 (1971).

${ }^{38}$ C. Merckling, M. El-Kazzi, G. Saint-Girons, G. Hollinger, L. Largeau, G. Patriarche, V. Favre-Nicolin, and O. Marty, J. Appl. Phys. 102, 024101 (2007).

${ }^{39}$ C. Merckling, M. El-Kazzi, V. Favre-Nicolin, M. Gendry, Y. Robach, G. Grenet, and G. Hollinger, Thin Solid Films 515, 6479 (2007).

${ }^{40}$ L. Qiao, T. C. Droubay, T. C. Kaspar, P. V. Sushko, and S. A. Chambers, Surf. Sci. 605, 1381 (2011).

${ }^{41}$ S. A. Chambers, L. Qiao, T. C. Droubay, T. C. Kaspar, B. W. Arey, and P. V. Sushko, Phys. Rev. Lett. 107, 206802 (2011).

${ }^{42}$ J. A. Rotole, Surf. Sci. Spectra 5, 18 (1998).

${ }^{43}$ Q. Fu and T. Wagner, Surf. Sci. Rep. 62, 431 (2007).

${ }^{44}$ V. A. Medvedev, D. D. Wagman, and J. D. Cox, CODATA Key Values for Thermodynamics (Hemisphere Publishing Corp., New York, 1989).

${ }^{45}$ J. M. McHale, A. Auroux, A. J. Perrotta, and A. Navrotsky, Science 277, 788 (1997). 